\title{
The Financial Development and Economic Growth: Based on the Data of City
}

\author{
Bin $\mathrm{Gu}^{1, \mathrm{a}}$, He Liur, ${ }^{2,{ }^{*}}$ \\ ${ }^{1}$ School of Economic and Commerce, South China University of Technology, \\ Guangzhou,Guangdong,China \\ ${ }^{2}$ School of Economic and Commerce, South China University of \\ Technology, Guangzhou,Guangdong,China \\ agubin@scut.edu.cn, b1093844805@qq.com
}

Keywords: The development of finance, The growth of economy, City.

\begin{abstract}
This paper used urban data as analysis object. The author collected data from 2003-2013, 288 cities in our country as the research foundation, using cross-section data regression of econometric model. This paper explored the correlation between financial development and economic, expounded the action mechanism of the two things in different regions. Then the author found that, from the national perspective, financial development promotes the economy, but the effect is not obvious. However, from the regional perspective, each region shows its unique characteristics. The impact is not so obvious as ideal. To some extent, financial development even inhibits the development of urban economy in some region. This reflects the current financial system still exists problems, which may be due to capital allocation, saving-investment transition rate, investment, insufficient investment quality, inefficient financial market and so on. Therefore, we should adjust the financial market and environment, carry out effective reforms of financial system, so as to make finance and economy mutually promotes each other and develop together.
\end{abstract}

\author{
金融发展与经济增长：基于城市数据的分析 \\ 谷斌1, a , 刘赫2,b, \\ 1华南理工大学管理科学与工程系, 广州, 广东, 中国 \\ 2华南理工大学管理科学与工程系, 广州, 广东, 中国 \\ agubin@scut.edu.cn, b1093844805@qq.com
}

关键词: 金融发展; 经济增长; 城市

中文摘要.本文以城市数据为分析对象，搜集了我国288个城市2003-2013的数据作为研究基 础, 运用了截面数据回归的计量模型, 对金融发展和经济增长的相关性进行了探究和实证, 阐述了不同区域二者作用的机制和作用的原因等。通过实证发现，全国的金融发展对经济有 一定的促进作用，但是效果并不明显。而进行区域的分析时，每个地区各有特征。金融中介 的发展对于经济提高的作用并不明显, 在某些区域甚至抑制了城市经济的发展。这反映出了 我国当前金融体系仍存在着一定问题, 可能是由于资本配置、储蓄投资转化率、投资质量不 足、金融市场运作低效等多种因素造成的。因此, 我们应调整金融市场以及金融环境, 对金 融体系进行有效的改革，让金融的发展和经济增长共同发展。 


\section{1. 引言}

近年来，伴随着世界经济的飞速发展和金融资产不断扩增，金融发展与经济增长的关系 受到广泛的关注。金融发展是促进经济增长的关键因素在一定程度上得到了经济界广泛的认 可。然而, 针对我国实际情况的研究工作还有很多提升空间, 金融发展和经济增长的具体内 在逻辑和影响机制都尚未被完全挖掘和揭露。总体来看, 对于金融发展和经济增长的研究在 我国已持续了一段时间, 也有逐步深入，不断充实的趋势。然而，我国的大部分文献是针对 中国整体为研究对象, 没有考虑到区域的差异性; 或以某个省域为对象, 不能得到全国性可借 鉴的结论。而少部分以区域数据进行实证的文献也存在着时间序列数据样本过少, 地区数量 不足的问题。尤其以城市数据为对象的实证研究还极为缺乏。另外, 我国的研究成果多数只 探讨了二者的相关性及因果关系, 对于具体内生因素以及内在逻辑的探讨都存在着研究空间。

于是，本文立足于中国国情，考虑到不同地区间经济和金融的发展不平衡性，本文选取 华北、东北、华东、华南、西北、华中、西南七大区域288个城市进行针对2003年到2013年的 城市金融发展和经济增长的数据的实证研究及分析。从而对两个对象因果行为的产生、强度 大小及具体机制、内在逻辑等多重角度进行全面的分析，从而立体地描述我国经济增长与金 融发展的联系，为调整优化我国经济结构、金融体系做奠基。

\section{2. 变量及检验方法的确定}

\section{1 指标的选择}

经济增长的指标选取了地区实际生产总值增长率, 即剔除了通货膨胀的影响的实际GDP 的增长率, 作为衡量经济增长的指标变量。

金融中介发展的指标选取了以存贷款为考量对象的两个指标进行回归分析，从而对我国 288 个城市金融发展的深度、规模等方面进行金融发展与经济增长的相关性的分析。衡量指标 如下:

（1）Credit是金融系统（银行机构和非银行金融机构）的总贷款与国民生产总值的比率, 衡量金融中介的整体深度。

（2）Deposit是金融系统中总存款相对于GDP的比例，是衡量金融中介整体规模的指标。

\section{2 检验方法的确定}

本文为了对于数据进行更全面的处理, 得出更符合实际发展情况的结论, 选择采用截面 数据分析的回归模型, 针对两个金融发展指标对变量进行回归, 从而分析其相关性和内在影 响机制。

\section{3. 数据描述及发展现状分析}

\section{1 数据来源及解释}

本文搜集整理了中国 288 个城市（直辖市 4 个，地级城市 284 个）的数据，把包括2003-20 13年的实际国内生产总值, 金融系统总贷款, 总存款, 进行处理、分析, 将这些城市划分为 华北、东北、华东、华南、西北、华中、西南七大区域, 将七大区域的金融发展和经济增长 的指标的回归结果进行比较, 从而得出各区域发展的差异, 深入验证金融发展和经济增长在 不同环境、不同制约因素下关系的变化和影响的程度变化。本文数据来自《中国城市统计年 鉴》, 中经网数据库。

\section{2 数据描述及现状分析}

为了更直观的描述各指标变量, 更清晰的表述区域之间的差异, 本文对不同区域的各项 指标以及所搜集的各类数据分别进行描述性统计。结果如下: 
表1（单位：亿元） GDP、总存款、总贷款描述性统计

\begin{tabular}{c|c|c|c|c|c|c|c}
\hline 均值 & 东北 & 华北 & 华东 & 华中 & 华南 & 西南 & 西北 \\
\hline GDP & 539.735 & 931.167 & 906.823 & 435.354 & 1007.409 & 467.118 & 282.702 \\
\hline 总存款 & 897.316 & 2541.843 & 1757.011 & 719.969 & 1783.073 & 919.143 & 638.386 \\
\hline 总贷款 & 608.087 & 1404.247 & 1259.553 & 5231.018 & 1137.296 & 672.796 & 417.308 \\
\hline
\end{tabular}

表2 GDP增速、credit、deposit描述性统计

\begin{tabular}{c|c|c|c|c|c|c|c}
\hline & 东北 & 华北 & 华东 & 华中 & 华南 & 西南 & 西北 \\
\hline GDP增速均值 & 13.645 & 13.487 & 14.187 & 12.655 & 14.466 & 13.976 & 13.386 \\
\hline GDP增速标准差 & 6.360 & 6.239 & 10.856 & 3.848 & 8.147 & 2.859 & 6.592 \\
\hline credit均值 & 0.937 & 1.243 & 1.104 & 0.962 & 1.230 & 1.123 & 1.135 \\
\hline credit标准差 & 0.429 & 0.580 & 0.509 & 0.496 & 1.560 & 0.646 & 0.761 \\
\hline deposit均值 & 1.475 & 2.113 & 1.478 & 1.383 & 1.477 & 1.664 & 1.795 \\
\hline deposit标准差 & 0.574 & 1.068 & 0.561 & 0.522 & 0.602 & 0.645 & 0.779 \\
\hline
\end{tabular}

可以看出, 各个区域存在着较大的落差。华南、华北、华东的地区生产总值相对较高, 而其他四个区域则显著低于前者, 说明华南、华北、华东在经济发展状况方面是处于全国领 先水平, 西部经济较为落后, 中部发展缓慢。而华南、华东两个地区GDP增速最高, 其他区域 则增长较为缓慢。可以看出, 生产总值增速和地区生产总值形势并不完全趋同, 华北地区虽 然经济水平较高, 但是增长速度并不理想。这也说明一些区域的经济发展可能处于相对懈急 的水平，并没有利用经济基础实行高效的经济发展策略。在存款方面，华北、华南、华东的 均值远高于其他区域, 与经济发展状况基本同步; 而华北、华东、华南的总贷款平均值高于 西南、东北、华中、西北，存贷款也具有同步的趋势。Deposit这个指标的描述显示，华北的 deposit均值要远高于其他区域, 而与经济发展不同的是, 西北、西南的deposit均值也相对 较高, 其次才是华东、华南、东北、华中。而华北、华南的Credit均值高于其他地区。在单 纯描述存款和贷款时, 各区域城市的统计数据与经济的发展步调基本相似, 然而当计算存贷 款占GDP的比重时，各个区域的发展情况则变得更为复杂也更有研究的价值。

根据以上对数据的描述, 我们可以看出我国金融发展和经济运行区域差异性是非常显著 的。发展上游城市和中下游城市水平落差十分明显, 华北、华南、华东这三个区域处于较发 达水平, 在各方面都有较迅速的发展, 这些主要得益于地理位置的优势以及政策性的倾向。

\section{4. 实证分析}

本文利用eviews8.0软件, 对全国以及不同区域的所有城市各指标的数据进行了截面数据 回归分析, 为了更全面精准的考虑到GDP增长速率的不同影响因素, 本文选取了控制变量如下: 一, 为了控制收玫效应对于经济增长的影响, 本文选取GDP的初始值作为解释变量与金融指标 同时回归, 控制城市初始规模对于经济增长的作用; 二, 本文应用产业结构比 (TSR), 即第三 产业占GDP的份额与第二产业占GDP的份额之比作为控制变量, 控制产业结构对于经济增长的 影响; 三, 为了反映城市固定资产投资对于经济增长的影响程度, 选取了固定资产投资比重 (IGR) 作为控制变量, 即固定资产投资份额与GDP的比率; 四, 考虑到工业发展对于经济增长 的影响, 本文的回归中解释变量中的控制变量也包含了工业总产值比重 (IDGR), 工业总产值 
与GDP之比。同时, 为了避免credit和deposit的变量自相关性对结果产生影响, 本文对两个 金融指标变量分别同以上控制变量对国民生产总值实际增速进行了回归。

首先从各区域城市混合的全国角度进行全部数据的两次回归，结果显示表3、4:

表3 全国Credit指标对GDP增速的回归结果

\begin{tabular}{c|c|c}
\hline 全国 & Coefficient & P值 \\
\hline Credit & 0.608079 & 0.1123 \\
\hline GDP & $-6.54 \mathrm{E}-08$ & 0.0905 \\
\hline TSR & 0.283795 & 0.5612 \\
\hline IGR & 2.796324 & 0.0057 \\
\hline IDGR & 0.402492 & 0.2673 \\
\hline $\mathrm{R}^{2}$ & \multicolumn{2}{c}{0.047464} \\
\hline Obs & \multicolumn{2}{c}{288} \\
\hline
\end{tabular}

表4 全国Deposit指标对GDP增速的回归结果

\begin{tabular}{c|c|c}
\hline 全国 & Coefficient & P值 \\
\hline Deposit & 0.536450 & 0.1014 \\
\hline GDP & $-4.05 \mathrm{E}-08$ & 0.2849 \\
\hline TSR & 0.481026 & 0.3148 \\
\hline IGR & 3.829668 & 0.0001 \\
\hline IDGR & 0.376622 & 0.3052 \\
\hline $\mathrm{R}^{2}$ & \multicolumn{2}{c}{0.050629} \\
\hline Obs & \multicolumn{2}{c}{288} \\
\hline
\end{tabular}

然后, 针对各个区域分别进行对于credit和deposit两个指标及控制变量进行对被解释变 量的两次回归。表5、6分别是credit和deposit的回归结果:

表5 credit作为解释变量的各区域回归结果

\begin{tabular}{c|c|c|c|c|c|c|c}
\hline & 东北 & 华北 & 华东 & 华中 & 华南 & 西南 & 西北 \\
\hline Credit & 2.450050 & -1.917511 & 1.330270 & 0.531807 & 0.371949 & 0.608687 & -0.274822 \\
\hline P值 & 0.0020 & 0.1366 & 0.1116 & 0.2269 & 0.6384 & 0.1953 & 0.6734 \\
\hline GDP & $-5.37 \mathrm{E}-08$ & $6.07 \mathrm{E}-08$ & $-1.20 \mathrm{E}-07$ & $4.36 \mathrm{E}-08$ & $-3.97 \mathrm{E}-08$ & $-3.12 \mathrm{E}-08$ & $-4.13 \mathrm{E}-08$ \\
\hline P值 & 0.5401 & 0.5989 & 0.0381 & 0.5863 & 0.6074 & 0.7038 & 0.8870 \\
\hline TSR & 0.432807 & -0.100018 & 0.428456 & -0.404097 & -1.517962 & 0.283136 & 0.448336 \\
\hline P值 & 0.6376 & 0.9661 & 0.7201 & 0.2311 & 0.2438 & 0.7347 & 0.8050 \\
\hline IGR & 4.133477 & 1.423387 & 3.282324 & -2.511444 & 13.25641 & 1.224223 & 1.451725 \\
\hline P值 & 0.0222 & 0.5777 & 0.1311 & 0.1157 & 0.0000 & 0.2256 & 0.4923 \\
\hline IDGR & 1.769982 & -1.570104 & 1.911453 & -1.594487 & -0.389293 & 1.049789 & -1.314961 \\
\hline P值 & 0.0222 & 0.5700 & 0.0561 & 0.0071 & 0.3985 & 0.3027 & 0.3515 \\
\hline R & 0.427621 & 0.136545 & 0.114769 & 0.134422 & 0.788875 & 0.043823 & 0.161476 \\
\hline DW值 & 2.007709 & 1.021968 & 2.096623 & 1.521497 & 1.725201 & 1.951980 & 1.153797 \\
\hline Obs & 34 & 33 & 78 & 42 & 37 & 34 & 30 \\
\hline
\end{tabular}

表6 deposit作为解释变量的各区域回归结果

\begin{tabular}{c|c|c|c|c|c|c|c}
\hline & 东北 & 华北 & 华东 & 华中 & 华南 & 西南 & 西北 \\
\hline Deposit & 1.105492 & -2.417516 & 1.441268 & 0.165991 & -0.741861 & 0.734072 & -0.362924 \\
\hline P值 & 0.2784 & 0.0024 & 0.0961 & 0.6737 & 0.3885 & 0.2077 & 0.6468 \\
\hline GDP & $-6.34 \mathrm{E}-08$ & $1.57 \mathrm{E}-07$ & $-1.30 \mathrm{E}-07$ & $6.38 \mathrm{E}-08$ & $-7.15 \mathrm{E}-09$ & $-4.39 \mathrm{E}-08$ & $-1.50 \mathrm{E}-08$ \\
\hline P值 & 0.5338 & 0.1440 & 0.0295 & 0.4344 & 0.9282 & 0.6161 & 0.9612 \\
\hline TSR & 0.867372 & -0.125521 & 0.202475 & -0.299474 & -1.030227 & 0.388714 & 0.369260 \\
\hline P值 & 0.4553 & 0.9507 & 0.8687 & 0.3728 & 0.4262 & 0.6368 & 0.8362 \\
\hline IGR & 5.293024 & 3.780054 & 3.043616 & -1.753761 & 13.92673 & 0.712001 & 1.844843 \\
\hline P值 & 0.0132 & 0.1185 & 0.1687 & 0.2381 & 0.0000 & 0.5638 & 0.4620 \\
\hline
\end{tabular}




\begin{tabular}{c|c|c|c|c|c|c|c}
\hline IDGR & 1.635064 & -2.065743 & 1.777651 & -1.532908 & -0.073278 & 1.116406 & -1.457929 \\
\hline $\mathrm{P}$ 值 & 0.0705 & 0.3835 & 0.0736 & 0.0111 & 0.8862 & 0.2708 & 0.3148 \\
\hline $\mathrm{R}^{2}$ & 0.223823 & 0.217396 & 0.117717 & 0.102582 & 0.792462 & 0.040316 & 0.162864 \\
\hline $\mathrm{DW}$ 值 & 1.827434 & 1.459722 & 2.069948 & 1.517736 & 1.934711 & 1.896936 & 1.163538 \\
\hline Obs & 34 & 33 & 78 & 42 & 37 & 34 & 30 \\
\hline
\end{tabular}

由截面数据回归的结果我们进行了分析以及说明，回归的结论如下:

从全国角度的分析结果来看, 我们采用的两个金融指标credit和deposit与经济增长指标 各城市实际地区生产总值增长率呈现一种不显著正相关关系, 说明全国的金融发展对于经济 的贡献并不高。同时, GDP与GDP实际增长率显示出负相关关系, 说明城市生产总值基数越大, 增长会相对缓慢。固定资产投资比重、产业结构比、工业总产值比重与经济增长速率都呈现 了正向的相关性，说明这些控制变量对于经济增长的作用的确是无法忽视的。

而从区域回归的结果来分析, 在对于credit指标的回归中, 大部分区域显现出一定的正 相关性。东北地区为系数最高的显著正相关; 其次, 华东、西南、华南是相对系数较低的正 相关; 华北和西北credit金融指标与经济增长率却显示为不显著的负相关。在关于deposit 指标的回归时, 同样, 大部分区域呈现正向相关, 华东地区相关系数最高; 其次东北, 西南 和华中则正相关系数偏低, 呈现不显著; 华北、华南、西北三个区域的存款指标与经济增长 为不显著的负相关。

这样的分析结果我们可以看出, 华北地区的两项存贷款指标均与经济增长指标呈现较强 负相关性, 该地区具有特殊性; 而西北地区也均呈现为较弱的负相关; 华南地区存款指标与 经济增长指标呈现了负相关。这说明, 当我们将研究的视角转到城市层面时, 金融对于经济 增长的促进作用其实是有限的。虽然在全国的宏观分析下，仍有一定的推进作用，但是各区 域并没有按预期实现金融发展对于经济增长的贡献性, 甚至可能抑制了该区域的发展。并且, 每个区域均有其影响特征, 无法得出一致的结论。这可能是由于区域的经济发展状况以及环 境、金融基础、政策倾向，银行运作机制等多种原因对区域的存贷款产生了不同的影响。

我们的研究结果在某种程度上与前人通过国家宏观数据或者省域数据研究的结果有些微 出入。但是细细勘酌, 似乎这样的结论也在某种意义上反映了在城市水平上某些区域经济增 长和经济发展的真实水平。虽然我国GDP近年来始终保持着高速增长的状态, 但是这种增长更 多的是依靠高投资, 而我国银行业投资的主要盈利模式是通过存贷款的利息差。高储蓄意味 着居民消费不足, 而消费是经济发展的主要动力, 这可能是导致金融对经济的作用甚微的重 要原因。我们根据马科 - 帕加洛的内生增长模型 (AK模型) 可以了解, 金融发展通过私人储蓄、 储蓄向投资转化率以及资本配置效率影响经济的增长。储蓄转化为投资的比率会影响到经济 的增长, 资本转化率越高, 资本倍增越快。而资本市场的发展, 银行体系的实际运行效率, 都能够影响储蓄向投资转化率。因此, 一方面高储蓄率影响了居民消费, 另一方面, 金融中 介体系的效率低下, 使得储蓄投资转化率低, 导致金融发展对经济增长在各区域的作用不明 显。再者, 根据投资收益递减理论, 当投资超过某临界值, 产生的收益率会开始下降, 这也 说明了，单单凭借金融资产数量的增长并不能真正让金融有效发展，并以此促进经济的进步。 投资效率、金融产品质量等的保障，才是能够证明金融发展有效性的基础和关键。

从以上分析可以看出, 我国当前城市金融的发展存在较多的问题, 金融体系也存在一定 的发展漏洞。区域之间的差异性严重影响了国家经济的同步发展以及经济政策的全面落实。 这些都使得在城市层面上, 金融发展对于经济增长的贡献极为有限。为了更好地发展经济, 我们不能限制金融的发展, 为了更好的达到金融和经济相互作用, 实现双赢, 最有效的措施 就是调整我国金融体系的模式和结构，并根据影响二者共同发展的因素进行完善。 


\section{5. 结束语}

金融作为当前经济发展的命脉, 有大量的研究成果表明其对经济发展有着重要的推动作 用。本文通过研究发现, 虽然在全国的分析结果来看, 金融促进经济发展。但是城市数据的 分析下，不同区域金融对于经济增长有促进也有抑制。在城市数据为研究对象时，我国金融 的发展并没有真正发挥出对经济增长的推进效果。我们需要改善培养金融投资环境，提高金 融中介及市场的运作效率，提供城市金融发展的设施和土壤，加强区域间合作交流，鼓励金 融产品的创新，从而引导我国金融发展和经济增长相互促进、共同发展。

\section{References}

[1] Li Yue. Study on the relationship between the financial development and economic growth in China - based on the provincial panel data analysis [D]. Jilin: Jilin university, 2014

[2] Qian Sen. The influence of financial development to economic growth research - in Yangtze river delta urban agglomeration [D]. Nanjing: science and technology of Nanjing university, 2009.

[3] Zeng Lin. Urban agglomeration of the relationship between financial development and economic growth based on the comparison of changzhutan and the pearl river delta [D]. Hunan: hunan normal university, 2013.

[4] Mao Shiqi. Financial development affects the regional difference of economic growth -- based on empirical deputy provincial cities under separate state planning variable coefficient model [D]. Ningbo, ningbo university, 2013

[5] Sun Lijun. The relationship between financial development and economic growth theory and empirical analysis [D]. Shanghai: Shanghai fudan university, 2007

[6]Tan Ruyong. The empirical study of relationship between China's financial development and economic growth [J]. Journal of economic research, 1999 (10) : 54-61

[7] Zhou Haowen,Zhong Yonghong. The development of Chinese financial intermediary and economic growth of regions: multivariate VAR system analysis [J]. Journal of gold Integrating research, 2004 (6) : 130-137

[8] Zhou Li,Wang Ziming. Empirical analysis on China's regional financial development and economic growth: 1978-2000 [J]. Journal of financial research Investigate, 2002 (10)

[9] Zhao Zhenquan, Xue Fenghui. An empirical analysis on the impact of financial development on economic growth [J]. Journal of financial research, 2004 (8):94-99

[10] Jiang Chun. Delay, Regression and preset: the empirical study of regional financial development and economic growth [J]. Journal of financial research, 2008 (02):198-206 